\title{
On Some Pursuit Differential Game Problem in a Hilbert Space
}

\author{
Jamilu Adamu, ${ }^{\text {a,* }}$, Aminu Sulaiman Halliru', Bala Ma'aji Abdulhamid ${ }^{\mathrm{c}}$ \\ ${ }^{a}$ Department of Mathematics, Federal University, Gashua, Nigeria. \\ ${ }^{b}$ Department of Mathematical Sciences, Bayero University, Kano, Nigeria. \\ ${ }^{c}$ Department of Mathematical Sciences, Abubakar Tafawa Balewa University, Bauchi, Nigeria.
}

\begin{abstract}
We study pursuit differential game problem in which a countable number of pursuers chase one evader. The problem is formulated in a Hilbert space $l_{2}$ with pursuers' motions described by $n^{\text {th }}$ order differential equations and that of the evader by $m^{\text {th }}$ order differential equation. The control functions of the pursuers and evader are subject to integral and geometric constraints respectively. Duration of the game is denoted by the positive number $\theta$. Pursuit is said to be completed if there exist strategies $u_{j}$ of the pursuers $P_{j}$ such that for any admissible control $v(\cdot)$ of the evader $E$ the inequality $\left\|y(\theta)-x_{j}(\theta)\right\| \leq r_{j}$ is satisfied for some $j \in\{1,2, \ldots\}$. In this paper, sufficient condition for completion of pursuit were obtained and also strategies of the pursuers that ensure completion of pursuit are constructed.
\end{abstract}

DOI:10.46481/jnsps.2022.379

Keywords: differential game, pursuer, evader, geometric constraint, integral constraint, Hilbert space.

Article History :

Received: 03 September 2021

Received in revised form: 25 January 2022

Accepted for publication: 25 January 2022

Published: 28 February 2022

(c)2022 Journal of the Nigerian Society of Physical Sciences. All rights reserved. Communicated by: T. Latunde

\section{Introduction}

A considerable amount of literature on differential game problem in which finite number of pursuers chase one evader in the Hilbert space $l_{2}$, control function of players subjected to either geometric, integral or both constraints has been published. (See for example, [1]- [25] and some reference their in.

In many studies of differential game problems, motions of the two players (i.e. pursuer and evader) are explicitly stated and are considered to be differential equations of the same order. For example, in the papers $[3,8,10,13,20,24]$, motion of each of the player is considered to obey first order differential

\footnotetext{
${ }^{*}$ Corresponding author tel. no: +2347033885836

Email address: jamiluadamu88@gmail.com (Jamilu Adamu )
}

equation. In other studies such as [5, 6, 17, 22], players' motions are described by second order differential equations. Whereas in $[1,2,4,21]$, motion of the pursuers and evader are described by first and second order differential equations respectively.

Adamu et. al [1], studied pursuit-evasion differential game problem in a Hilbert space $l_{2}$, in which motions of pursuers and evader are described by first and second order differential equations respectively. Control functions of both the pursuers and evader are subject to integral constrains. Under certain condition the authors found the value of the game and constructed optimal strategies of the players.

In the paper [2] by Badakaya, a pursuit differential game problem with finite number of pursuers and one evader in the space $l_{2}$ is considered. Pursuers' motions are described 
by a first order differential equations and that of the evader by a second order differential equation. Control functions of the pursuers and evader are subject to integral and geometric constraints respectively. Duration of the game is denoted by the positive number $\theta$. Theorems are stated and proved each of which provides a condition for completion of pursuit. Consequently, strategies of the pursuers that ensure completion of pursuit are constructed.

In the other hand, the paper [4], is about study of pursuit differential game problem in 1-catch sense with finite number of pursuers and one evader in the space $l_{2}$. In this paper, control functions of the pursuers and evader are subject to integral and geometric constraints respectively. Sufficient condition for completion of pursuit were obtained.

Leong and Ibragimov [12] studied simple motion pursuit differential game with $m$ pursuers and one evader on a closed convex subset of the Hilbert space $l_{2}$. Control functions of the players are subjected to integral constraints. The total resource of the pursuers is assumed to be greater than that of the evader. Strategy of pursuers were constructed sufficient to complete the pursuit from any initial position.

In the paper [20], simple motion differential game with many players and geometric constraints on the control functions of the players was studied. By using Lyapunov function method for an auxiliary problem, they obtained sufficient conditions to find the pursuit time in $R^{n}$.

The paper [21] deal with the study of a pursuit differential game problem for the so-called "boy and crocodile" game in the space $R^{n}$. Boy's motion is described by a first order differential equations and that of the crocodile by a second order differential equation. Control functions of the pursuer and evader are subject to integral and geometric constraints respectively. They obtained sufficient conditions of completion of pursuit.

Vagin and Petrov [25] studied a pursuit differential game problem with finite number pursuers and evader in the space $R^{n}$. Motions of the players are described by $n^{\text {th }}$ order differential equations. Control functions of the players are subject to geometric constraints. Sufficient condition for completion of pursuit was obtained.

In the present paper, motivated by the above development, we proposed to study pursuit differential game problem with countable number of pursuers and one evader in the Hilbert space $l_{2}$. The motions of the pursuers and evader are described by $n^{\text {th }}$ and $m^{\text {th }}$ order differential equations respectively. Control functions of the pursuers are subject to integral constrains. Whereas, geometric constraint is imposed on the control function of the evader. We found the sufficient condition of completion of pursuit in 1-catch sense.

\section{Differential game formulation}

Let $l_{2}=\left\{\xi=\left(\xi_{1}, \xi_{2}, \ldots\right): \sum_{j=1}^{\infty} \xi_{j}^{2}<\infty\right\}$, with inner product $\langle\cdot, \cdot\rangle: l_{2} \times l_{2} \rightarrow R$ and norm $\|\cdot\|: l_{2} \rightarrow[0,+\infty)$, defined as follows:

$$
\langle\xi, y\rangle=\sum_{j=1}^{\infty} \xi_{j} y_{j}, \quad\|z\|=\left(\sum_{j=1}^{\infty} z_{j}^{2}\right)^{1 / 2},
$$

where $\xi, y, z \in l_{2}$.

We consider a differential game described by the following initial value problem:

$$
\left\{\begin{array}{c}
P_{j}: \frac{d^{n} x_{j}}{d t^{n}}=u_{j}(t) \\
E: \frac{d^{m} y}{d t^{m}}=v(t)
\end{array}\right.
$$

subject to:

$$
\left\{\begin{array}{l}
x_{j}(0)=x_{j}^{0}, \frac{d x_{j}}{d t}(0)=x_{j}^{1}, \frac{d^{2} x_{j}}{d t^{2}}(0)=x_{j}^{2}, \ldots, \frac{d^{n-1} x_{j}}{d t^{n-1}}(0)=x_{j}^{n-1}, j \in J, \\
y(0)=y^{0}, \frac{d y}{d t}(0)=y^{1}, \frac{d^{2} y}{d t^{2}}(0)=y^{2}, \ldots, \frac{d^{m-1} y}{d t^{m-1}}(0)=y^{m-1}
\end{array}\right.
$$

where $x_{j}, x_{j}^{0}, x_{j}^{1}, x_{j}^{2}, \ldots, x_{j}^{n-1}, u_{j}, y, y^{0}, y^{1}, y^{2}, \ldots, y^{m-1}, v \in l_{2}$, $u_{j}$ and $v$ are controls parameter of the pursuer $P_{j}$ and evader $E$ respectively. Here and below $n \leq m$ for all $n, m \in N$, $J=\{1,2, \ldots\}$ and $\left\|y_{0}-x_{j 0}\right\|>r_{j}$, where $r_{j} \geq 0$.

Let $C\left(0, \theta ; l_{2}\right)$ be the space of functions

$$
h(t)=\left(h_{1}(t), h_{2}(t), \cdots, h_{j}(t), \cdots\right) \in l_{2}, \quad t \geq 0,
$$

such that the following conditions hold:

(a) $h_{j}(t), 0 \leq t \leq \theta, \quad j=1,2, \cdots$, are absolutely continuous functions;

(b) $h(t), 0 \leq t \leq \theta$, is a continuous function in the norm of $l_{2}$.

Definition 2.1. A function $u_{j}(t)=\left(u_{j 1}(t), u_{j 2}(t), \ldots\right)$ with Borel measurable coordinates such that

$$
\int_{0}^{\theta}\left\|u_{j}(t)\right\|^{2} d t \leq \beta_{j}^{2}
$$

where $\beta_{j}$ is given positive number, is called an admissible control of the $j^{\text {th }}$ pursuer.

Definition 2.2. A function $v(t)=\left(v_{1}(t), v_{2}(t), \ldots\right)$ with Borel measurable coordinates such that

$$
\|v(t)\| \leq \gamma, \quad t \geq 0
$$

where $\gamma$ is given positive number, is called admissible control of the evader $E$.

Definition 2.3. A function $U_{j}\left(t, x_{j}, y, v\right), U_{j}:[0, \theta] \times l_{2} \times l_{2} \times$ $l_{2} \rightarrow l_{2}$, is called a strategy of the $j^{\text {th }}$ pursuer if, for any admissible control $v(\cdot)$ of the evader $E$, the system (1)-(2) has a unique solution $\left(x_{j}(\cdot), y(\cdot)\right)$, with $x_{j}(\cdot), y(\cdot) \in C\left(0, \theta, l_{2}\right)$. A strategy $U_{j}$ is admissible if each control involved in the formation of this strategy is admissible. 
Definition 2.4. The system described by (1)-(2) in which the controls $u_{j}(\cdot)$ and $v(\cdot)$ satisfy the inequalities (4) and (5) respectively is called game $G_{1}$.

Definition 2.5. Pursuit is said to be completed in l-catch sense in the game $G_{1}$ if there exist strategies $u_{j}$ of the pursuers $P_{j}$ such that for any admissible control $v(\cdot)$ of the evader $E$ the inequality $\left\|y(\theta)-x_{j}(\theta)\right\| \leq r_{j}$ is satisfied for some $j \in\{1,2, \ldots\}$.

Research problem: In the game $G_{1}$, find sufficient condition for completion of pursuit.

\section{MAIN RESULT}

Once the players' admissible controls $u_{j}(\cdot)$ and $v(\cdot)$ are chosen, the solution to the equations of motion for the $j^{\text {th }}$ pursuer and evader in (1) with initial condition (2) are respectively given by

$$
\begin{aligned}
& x_{j}(\theta)=x_{j 0}+\int_{0}^{\theta} \int_{0}^{t_{1}} \int_{0}^{t_{2}} \cdots \int_{0}^{t_{n-1}} u_{j}(t) d t d t_{n-1} \\
& \ldots d t_{2} d t_{1},
\end{aligned}
$$

and

$y(\theta)$

$$
=y_{0}+\int_{0}^{\theta} \int_{0}^{t_{1}} \int_{0}^{t_{2}} \cdots \int_{0}^{t_{m-1}} v(t) d t d t_{m-1} \ldots d t_{1},(7)
$$

where

$$
\begin{aligned}
& x_{j 0}=x_{j}^{0}+\theta x_{j}^{1}+\frac{\theta^{2}}{2 !} x_{j}^{2}+\cdots+\frac{\theta^{n-1}}{(n-1) !} x_{j}^{n-1} \\
& y_{0}=y^{0}+\theta y^{1}+\frac{\theta^{2}}{2 !} y^{2}+\cdots+\frac{\theta^{m-1}}{(m-1) !} y^{m-1}
\end{aligned}
$$

The expression with the multiple integrals in (6) and (7) can be reduced to that with single integral using the formula below (see [27])

$$
\begin{array}{r}
\int_{0}^{\theta} \int_{0}^{t_{1}} \int_{0}^{t_{2}} \cdots \int_{0}^{t_{n-1}} u(t) d t_{n-1} \ldots d t_{2} d t_{1} \\
=\int_{0}^{\theta} \frac{(\theta-t)^{n-1}}{(n-1) !} u(t) d t .
\end{array}
$$

Therefore, equation (6) and (7) become

$$
\begin{aligned}
& x_{j}(\theta)=x_{j 0}+\int_{0}^{\theta} \frac{(\theta-t)^{n-1}}{(n-1) !} u_{j}(t) d t, \\
& y(\theta)=y_{0}+\int_{0}^{\theta} \frac{(\theta-t)^{m-1}}{(m-1) !} v(t) d t,
\end{aligned}
$$

Alternatively, in place of the players' dynamic equations (1), we can consider an equivalent differential game described by the following first order differential equations:

$$
\left\{\begin{array}{l}
P_{j}: \frac{d x_{j}}{d t}=\frac{(\theta-t)^{n-1}}{(n-1) !} u_{j}(t), x_{j}(0)=x_{j 0}, \\
E: \frac{d y}{d t}=\frac{(\theta-t)^{m-1}}{(m-1) !} v(t), y(0)=y_{0},
\end{array}\right.
$$

Let $\pi_{1}=\omega_{0}\left(\beta_{j}^{2}-\gamma^{2} \omega_{1}\right)+\left\|y_{0}\right\|^{2}-\left\|x_{j 0}\right\|^{2}$ and $\pi_{2}=\omega_{0}\left(\beta_{j}^{2}-\theta \gamma^{2}\right)+\left\|y_{0}\right\|^{2}-\left\|x_{j 0}\right\|^{2}$, where $\omega_{0}=\frac{\theta^{2 n-1}}{(2 n-1)((n-1) !)^{2}}$ and $\omega_{1}=\frac{((n-1) !)^{2} \theta^{2(m-n)+1}}{(2(m-n)+1)((m-1) !)^{2}}$.

We define the sets

$$
X:=\left\{\begin{array}{l}
\bigcup_{j \in J}\left\{z \in l_{2}: 2\left\langle y_{0}-x_{j 0}, z\right\rangle \leq \pi_{1}\right\}, n<m . \\
\bigcup_{j \in J}\left\{z \in l_{2}: 2\left\langle y_{0}-x_{j 0}, z\right\rangle \leq \pi_{2}\right\}, n=m .
\end{array}\right.
$$

The following statement gives sufficient condition for completion of pursuit.

Theorem 3.1. . If $y(\theta) \in X$ then the pursuit can be completed in the game $G_{1}$.

Proof. To prove this theorem, we first introduce dummy pursuer with state variable $z$ whose motion obey the following initial value problem.

$$
\frac{d z}{d t}=\frac{(\theta-t)^{n-1}}{(n-1) !} \bar{U}(t), \quad z(0)=z_{0}=x_{j 0},
$$

and that the control function $\bar{U}(\cdot)$ is such that

$$
\left(\int_{0}^{\theta}\|\bar{U}(t)\|^{2} d t\right)^{\frac{1}{2}} \leq \bar{\beta}=\beta_{j}+\frac{r_{j}}{\sqrt{\omega_{0}}}
$$

Clearly, $\bar{\beta}>\beta_{j}$ and $\left(\bar{\beta}-\beta_{j}\right) \sqrt{\omega_{0}}=r_{j}$ for all $j \in J$.

For all $j \in J$ and $0 \leq t \leq \theta$, we construct a dummy pursuer's strategy as follows: If $n=m$, we set

$$
\bar{U}(t)=\frac{(2 n-1)(n-1) !}{\theta^{2 n-1}(\theta-t)^{1-n}}\left(y_{0}-z_{0}\right)+v(t) .
$$

If $n<m$, we set

$$
\bar{U}(t)=\frac{(2 n-1)(n-1) !}{\theta^{2 n-1}(\theta-t)^{1-n}}\left(y_{0}-z_{0}\right)+\frac{(n-1) ! v(t)}{(m-1) !(\theta-t)^{n-m}} .
$$

For the case $n=m$, let the dummy pursuer $z$ use the strategy (17), we show that this strategy is admissible and ensure the equality $z(\theta)=y(\theta)$. Indeed,

$$
\begin{aligned}
z(\theta) & =z_{0}+\int_{0}^{\theta} \frac{(\theta-t)^{n-1}}{(n-1) !} \\
& \times\left(\frac{(2 n-1)(n-1) !}{\theta^{2 n-1}(\theta-t)^{1-n}}\left(y_{0}-x_{j 0}\right)+v(t)\right) d t \\
& =z_{0}+\frac{(2 n-1)\left(y_{0}-x_{j 0}\right)}{\theta^{2 n-1}} \int_{0}^{\theta}(\theta-t)^{2 n-2} d t \\
& +\int_{0}^{\theta} \frac{(\theta-t)^{n-1}}{(n-1) !} v(t) d t \\
& =z_{0}+y_{0}-x_{j 0}+\int_{0}^{\theta} \frac{(\theta-t)^{n-1}}{(n-1) !} v(t) d t \\
& =y_{0}+\int_{0}^{\theta} \frac{(\theta-t)^{m-1}}{(m-1) !} v(t) d t=y(\theta) .
\end{aligned}
$$


It is left to show that the strategy (17) is admissible. By assumption $y(\theta) \in X$ and if $n=m$, we have,

$$
2\left\langle y_{0}-x_{j 0}, y(\theta)\right\rangle \leq \omega_{0}\left(\beta_{j}^{2}-\theta \gamma^{2}\right)+\left\|y_{0}\right\|^{2}-\left\|x_{j 0}\right\|^{2}
$$

In accordance with inequality (19) and using state equation (12) of the evader, we have:

$$
\begin{array}{r}
2\left\langle y_{0}-x_{j 0}, \int_{0}^{\theta} \frac{(\theta-t)^{m-1}}{(m-1) !} v(t) d t\right\rangle \\
\quad \leq \omega_{0}\left(\beta_{j}^{2}-\theta \gamma^{2}\right)-\left\|y_{0}-x_{j 0}\right\|^{2} .
\end{array}
$$

Using the inequality (20), we obtained

$$
\begin{aligned}
\int_{0}^{\theta} & \|\bar{U}(t)\|^{2} d t \\
& =\int_{0}^{\theta}\left\|\frac{(2 n-1)(n-1) !}{\theta^{2 n-1}(\theta-t)^{1-n}}\left(y_{0}-z_{0}\right)+v(t)\right\|^{2} d t \\
& =\int_{0}^{\theta}\left\|\frac{(2 n-1)(n-1) !}{\theta^{2 n-1}(\theta-t)^{1-n}}\left(y_{0}-x_{j 0}\right)\right\|^{2} d t \\
& +2 \int_{0}^{\theta}\left\langle\frac{(2 n-1)(n-1) !}{\theta^{2 n-1}(\theta-t)^{1-n}}\left(y_{0}-x_{j 0}\right), v(t)\right\rangle d t \\
& +\int_{0}^{\theta}\|v(t)\|^{2} d t \\
\leq & \frac{\left\|y_{0}-x_{j 0}\right\|^{2}}{\omega_{0}}+\frac{2}{\omega_{0}}\left\langle y_{0}-x_{j 0}, \int_{0}^{\theta} \frac{(\theta-t)^{m-1}}{(m-1) !} v(t) d t\right\rangle \\
+ & \theta \gamma^{2} \\
\leq & \left\|y_{0}-x_{j 0}\right\|^{2} \\
+ & \theta \gamma^{2} \\
& =\beta_{j}^{2}<\bar{\beta}^{2}
\end{aligned}
$$

For the case $n<m$ and if the dummy pursuer $z$ uses the strategy (18), we have $z(\theta)=y(\theta)$. Indeed, clearly

$$
\begin{aligned}
z(\theta) & =x_{j 0}+\frac{(2 n-1)\left(y_{0}-x_{j 0}\right)}{\theta^{2 n-1}} \int_{0}^{\theta}(\theta-t)^{2 n-2} d t \\
& +\int_{0}^{\theta} \frac{(\theta-t)^{m-1}}{(m-1) !} v(t) d t \\
& =x_{j 0}+\frac{(2 n-1)\left(y_{0}-x_{j 0}\right)}{\theta^{2 n-1}} \frac{\theta^{2 n-1}}{2 n-1} \\
& +\int_{0}^{\theta} \frac{(\theta-t)^{m-1}}{(m-1) !} v(t) d t \\
& =x_{j 0}+y_{0}-x_{j 0}+\int_{0}^{\theta} \frac{(\theta-t)^{m-1}}{(m-1) !} v(t) d t \\
& =y_{0}+\int_{0}^{\theta} \frac{(\theta-t)^{m-1}}{(m-1) !} v(t) d t=y(\theta) .
\end{aligned}
$$

The admissibility of the strategy (18) can be shown as follows. The inclusion $y(\theta) \in X$, implies that,

$$
2\left\langle y_{0}-x_{j 0}, y(\theta)\right\rangle \leq \omega_{0}\left(\beta_{j}^{2}-\gamma^{2} \omega_{1}\right)+\left\|y_{0}\right\|^{2}-\left\|x_{j 0}\right\|^{2}
$$

In accordance with inequality (21) and using state equation (12) of the evader, we have:

$$
\begin{aligned}
& 2\left\langle y_{0}-x_{j 0}, \int_{0}^{\theta} \frac{(\theta-t)^{m-1}}{(m-1) !} v(t) d t\right\rangle \\
& \leq \omega_{0}\left(\beta_{j}^{2}-\gamma^{2} \omega_{1}\right)-\left\|y_{0}-x_{j 0}\right\|^{2} .
\end{aligned}
$$

Using the inequality (22), we obtained

$$
\begin{aligned}
& \int_{0}^{\theta}\|\bar{U}(t)\|^{2} d t \\
& =\int_{0}^{\theta}\left\|\frac{(2 n-1)(n-1) !}{\theta^{2 n-1}(\theta-t)^{1-n}}\left(y_{0}-x_{j 0}\right)+\frac{(n-1) ! v(t)}{(m-1) !(\theta-t)^{n-m}}\right\|^{2} d t \\
& =\int_{0}^{\theta}\left\|\frac{(2 n-1)(n-1) !}{\theta^{2 n-1}(\theta-t)^{1-n}}\left(y_{0}-x_{j 0}\right)\right\|^{2} d t \\
& +2 \int_{0}^{\theta}\left\langle\frac{(2 n-1)(n-1) !}{\theta^{2 n-1}(\theta-t)^{1-n}}\left(y_{0}-x_{j 0}\right), \frac{(n-1) ! v(t)}{(m-1) !(\theta-t)^{n-m}}\right\rangle d t \\
& +\int_{0}^{\theta}\left\|\frac{(n-1) ! v(t)}{(m-1) !(\theta-t)^{n-m}}\right\|^{2} d t \\
& \leq \frac{\left\|y_{0}-x_{j 0}\right\|^{2}}{\omega_{0}}+\frac{2}{\omega_{0}}\left\langle y_{0}-x_{j 0}, \int_{0}^{\theta} \frac{(\theta-t)^{m-1}}{(m-1) !} v(t) d t\right\rangle+\gamma^{2} \omega_{1} \\
& \leq \frac{\left\|y_{0}-x_{j 0}\right\|^{2}}{\omega_{0}}+\frac{1}{\omega_{0}}\left(\omega_{0}\left(\beta_{j}^{2}-\gamma^{2} \omega_{1}\right)-\left\|y_{0}-x_{j 0}\right\|^{2}\right)+\gamma^{2} \omega_{1} \\
& =\beta_{j}^{2}<\bar{\beta}^{2}
\end{aligned}
$$

This shows that for each two cases we established that $z(\theta)=$ $y(\theta)$. With this and if the real pursuers use the strategies

$$
U_{j}(t)=\frac{\beta_{j}}{\bar{\beta}} \bar{U}(t), 0 \leq t \leq \theta,
$$

we show that

$$
\left\|y(\theta)-x_{j}(\theta)\right\| \leq r_{j}
$$

Indeed, using equation (22) and Cauchy-Schwartz inequality, we get

$$
\begin{aligned}
\| y & (\theta)-x_{j}(\theta)\|=\| z(\theta)-x_{j}(\theta) \| \\
& =\left\|z_{0}+\int_{0}^{\theta} \frac{(\theta-t)^{n-1}}{(n-1) !} \bar{U}(t) d t-x_{j 0}-\int_{0}^{\theta} \frac{(\theta-t)^{n-1}}{(n-1) !} U_{j}(t) d t\right\| \\
& =\left\|\int_{0}^{\theta} \frac{(\theta-t)^{n-1}}{(n-1) !}\left(\bar{U}(t)-\frac{\beta_{j}}{\bar{\beta}} \bar{U}(t)\right) d t\right\| \\
& \leq \int_{0}^{\theta}\left\|\frac{(\theta-t)^{n-1}}{(n-1) !}\left(1-\frac{\beta_{j}}{\bar{\beta}}\right) \bar{U}(t)\right\| d t \\
& =\left(\frac{\bar{\beta}-\beta_{j}}{\bar{\beta}}\right) \int_{0}^{\theta} \frac{(\theta-t)^{n-1}}{(n-1) !}\|\bar{U}(t)\| d t \\
& \leq\left(\frac{\bar{\beta}-\beta_{j}}{\bar{\beta}}\right)\left[\left(\int_{0}^{\theta} \frac{(\theta-t)^{2 n-2}}{((n-1) !)^{2}} d t\right)^{\frac{1}{2}}\left(\int_{0}^{\theta}\|\bar{U}(t)\|^{2} d t\right)^{\frac{1}{2}}\right] \\
& \leq\left(\frac{\bar{\beta}-\beta_{j}}{\bar{\beta}}\right)\left(\frac{\theta^{2 n-1}}{(2 n-1)((n-1) !)^{2}}\right)^{\frac{1}{2}} \bar{\beta} \\
& \leq\left(\bar{\beta}-\beta_{j}\right) \sqrt{\omega_{0}}=r_{j} .
\end{aligned}
$$

This complete the prove of the theorem. 


\section{Conclusion}

We studied pursuit differential game problem in which a countable number of pursuers chase one evader in the Hilbert space $l_{2}$. Control function of the pursuers and evader are subject to integral and geometric constraints respectively. Pursuers' motions are described by $n^{\text {th }}$ order differential equations and that of the evader by $m^{\text {th }}$ order differential equation where $n \leq m, n, m \in N$. In this piece of research the strategies of the pursuers are constructed and sufficient condition for completion of pursuit were obtained. The result of this paper is the generalization of some result in the literature. For example, the result of the paper [4] is a corrolary to this paper when we set $n=1$ and $m=2$ in the players' dynamic equation (1)-(2).

\section{Acknowledgements}

The authors would like to thank the referee for giving useful comments and suggestions for improvement of this paper.

\section{References}

[1] J. Adamu, K. Muangehoo, A. J. Badakaya and J. Rilwan, "On pursuitevasion differential game problem in a Hilbert space", AIMS mathematics 5 (2020) 7467.

[2] A. J Badakaya, "A Pursuit Differential Game Problems with Different Constraints on the Control of the Players", Transaction of the Nigerian Association of Mathematical Physics 8 (2019) 17.

[3] G. I. Ibragimov and B. B. Rikhsiev, "On some Sufficient Conditions for Optimalty of the Pursuit Time in the Differential Game with Multiple Pusuers", Automation and Remote Control 67 (2006) 529.

[4] J. Adamu, B. M. Abdulhamid, D. T. Gbande and A. S. Halliru, "Simple motion pursuit differential game", Journal of the Nigerian Society Physical Sciences 3 (2021) 12.

[5] G. I. Ibragimov and N. A. Hussin, "A Pursuit-Evasion Differential Game with Many Pursuers and One Evader", Malaysian Journal of Mathematical Sciences 4 (2010) 183.

[6] G. I. Ibragimov and M. Salimi, "Pursuit-Evasion Differential Game with Many Inertial Players", Mathematical Problems in Engineering 2009 (2009) 15.

[7] G. I. Ibragimov, A. Sh. Kuchkarov, "Fixed Duration Pursuit-Evasion Differential Game with Integral Constraints", Journal of Physics Conference Series 435 (2017).

[8] G. I. Ibragimov, "Optimal pursuit with Countably Many Pursuers and one Evader", Differential Equations 41 (2005) 627.

[9] G. I. Ibragimov, "Simple motion pursuit differential game of many pursuers and one evader on convex compact set", International Journal of pure and Applied Mathematics 4 (2015) 733.
[10] G. I. Ibragimov and N. Satimov, "A Multiplayer Pursuit Differential Game on a Convext Set with Integral Constraints", Abstract and Applied Analysis, 2012 (2012) doi: 10.1155/2012/460171.

[11] G. I. Ibragimov, and A. I. Alias, "A pursuit problem described by infinite system of differential equations with coordinate-wise integral constraints on control functions", Malaysian Journal of Applied Mathematical Sciences 9 (2015) 67.

[12] W. J. Leong, and G. I. Ibragimov, "A multiperson pursuit problem on a closed convex set in Hilbert spaces", Far East Journal of Applied Mathematics, 33 (2008) 205.

[13] A. Kuchkarov, G. I. Ibragimov and M. Ferrara, "Simple Motion Pursuit and Evasion Differential Games with Many Pursuers on Manifolds with Euclidean Metric", Discrete Dynamics in Nature and Society 2016 (2016) http://dx.doi.org/10.1155/2016/1386242.

[14] A. B. Jaafaru and G. I. Gafurjan, "On Some Pursuit and Evasion Differential Game Problems for an Infinite Number of First-Order Differential Equations", Journal of Applied Mathematics 2012 (2012) 13.

[15] A. B. Jaafaru and G. I. Gafurjan, "Differential Game described by Infinite system of DE'S of $2^{\text {nd }}$ Order. The case of negetive coefficients", International Journal of Pure and Applied Mathematics 70 (2011) 927.

[16] M. Salimi, G. I. Ibragimov, S. Stefen and S. Somayeh, "On a fixed duration Pursuit differential game with geometric and integral constraints", Dyn Games Appl. 2015 (2015) doi.org/10.1007/s 13235-015-0161-3.

[17] G. I. Ibragimov, N. Abd Rashid, A. Kuchkarov and F. Ismail," Multi Pursuer Differential Game of Optimal Approach with Integral Constraints on Controls of the Players", Taiwanese Journal of Mathematics 19 (2015) 963.

[18] G. I. Ibragimov, "A Game of Optimal Pursuit of One Object by Several", Journal of Applied Mathematics and Mechanics 62 (1988) 187.

[19] G. I. Ibragimov, "Game Problem on a closed convex set", Siberian Advances in Mathematics 12 (2002) 16.

[20] R. P. Ivanov and Yu. S. Ledyaev, "Optimality of pursuit time in a simple motion differential game of many objects", Trudy Matematicheskogo Instituta imeni V. A. Steklova 158 (1981) 87.

[21] N. Siddiqoba, S. Muksimova, A. Rakhmanov," Research of One Problem of Different Constraints on the Controls", International Journal of Scientific and Research Publications 7 (2017) 65.

[22] B. T. Samatov," Problems of Group Pursuit with Integral Constraints on Controls of the Players" I, Cybernetics and System Analysis 49 (2013) 756.

[23] M. Salimi and M. Ferrara, "Differential Game of Optimal Pursuit of one Evader by Many Pursuers", International Journal of Game Theory 48 (2019) 481.

[24] A. YU. Levchenkov and A. G. Pashkov, "Differential Game of Optimal Approach of Two Inertial Pursuers to a Noninertial Evader", Journal of Optimization Theory and Applications 65 (1990) 501.

[25] D. A. Vagin and N. N. Petrov, "A Problem of Group Pursuit with Phase Constraints", Journal of Applied Mathematics and Mechanics 66 (2002) 225.

[26] A. I. Subbotin, and A. G. Chentsov, Optimazition of Guaranteed Result in Control Problem, Moscow, Russia, 1981.

[27] W. Abdul-majid, Linear and Nonlinear Integral Equation Methods and Applications, 10-20, Saints Xavier University, Chicago , 11,60655, USA, 2011. 